\title{
Recurrent brainstem cavernous malformations following primary resection: blind spots, fine lines, and the right-angle method
}

\author{
Roxanna M. Garcia, MD, MS, MPH, ${ }^{1}$ Taemin Oh, MD, ${ }^{2}$ Tyler S. Cole, MD, ${ }^{1}$ \\ Benjamin K. Hendricks, MD, ${ }^{1}$ and Michael T. Lawton, MD1 \\ 1Department of Neurosurgery, Barrow Neurological Institute, St. Joseph's Hospital and Medical Center, Phoenix, Arizona; and \\ ${ }^{2}$ Department of Neurological Surgery, University of California, San Francisco, California
}

\begin{abstract}
OBJECTIVE Proximity of brainstem cavernous malformations (BSCMs) to tracts and cranial nerve nuclei make it costly to transgress normal tissue in accessing the lesion or disrupting normal tissue adjacent to the lesion in the separation plane. This interplay between tissue sensitivity and extreme eloquence makes it difficult to avoid leaving a remnant on occasion. Recurrences require operative intervention, which may increase morbidity, lengthen recovery, and add to overall costs. An approximately 20-year experience with patients with recurrent BSCM lesions following primary microsurgical resection was reviewed.
\end{abstract}

METHODS A prospectively maintained database of 802 patients who underwent microsurgical resection of cerebral cavernous malformations during 1997-2018 was queried to identify 213 patients with BSCMs. A retrospective chart review was conducted for patients with recurrent BSCM after primary resection who required a second surgery.

RESULTS Fourteen of 213 patients (6.6\%) underwent repeat resection for recurrent BSCM. Thirty-four hemorrhagic events were observed among these 14 patients over 576 patient-years (recurrent hemorrhage rate, $5.9 \%$ per year; median discrete hemorrhagic events, 2; median time to rehemorrhage, 897 days). BSCM occurred in the pons in 10 cases, midbrain in 2 cases, and medulla in 2 cases. A blind spot in the operative corridor was the most common cause of residual BSCM (9 patients). All recurrent BSCMs were removed completely, although 2 patients each required 2 operations to treat recurrence. Twelve patients had unchanged or improved modified Rankin Scale scores at last clinical evaluation compared with admission, and 2 patients had worse scores. Recurrence was more common among patients who were operated on in the first versus the second half of the series ( $8.5 \%$ vs $4.7 \%$ ).

CONCLUSIONS The $6.6 \%$ rate of BSCM recurrence requiring reoperation reflects the fine lines between complete resection and recurrence and between safe and harmful surgery. The detection of remnants is difficult postoperatively and remains so even at 6 months when the resection bed has healed. The $5.9 \%$ annual hemorrhage risk associated with recurrent BSCM in this experience is consistent with that reported for unoperated BSCMs. The right-angle method helps to anticipate blind spots and meticulously inspect the resection cavity for residual BSCM during surgery. A low percentage of recurrent BSCM $(5 \%-10 \%)$ ensures ongoing effort toward an acceptable balance of safety and completeness.

https://thejns.org/doi/abs/10.3171/2020.6.JNS201555

KEYWORDS brainstem cavernous malformation; cavernoma; recurrent; remnant; reoperation; residual; vascular disorders

$\mathrm{E}$ VOLVING surgical perspectives have transformed the brainstem cavernous malformation (BSCM) from an inoperable lesion in a surgical no man's land to a lesion that is best managed microsurgically using skull base approaches and the lesion's presentation on pial or ependymal surfaces to access it safely while avoiding neural tracts and cranial nerve $(\mathrm{CN})$ nuclei. Several system- atic reviews of BSCMs describing the natural history and management of these lesions ${ }^{1-3}$ have established microsurgical resection as the standard of care following symptomatic hemorrhage within the context of the patient's clinical status and life expectancy, the lesion's accessibility within the brainstem, and the estimated risk of rehemorrhage. ${ }^{4,5}$ Gross et al. ${ }^{2}$ reported an overall $91 \%$ complete resection

ABBREVIATIONS BSCM = brainstem cavernous malformation; $\mathrm{CN}=$ cranial nerve; $\mathrm{mRS}=$ modified Rankin Scale

SUBMITTED April 30, 2020. ACCEPTED June 30, 2020.

INCLUDE WHEN CITING Published online November 20, 2020; DOI: 10.3171/2020.6.JNS201555. 
rate among 1390 patients aggregated from the literature, with rates ranging from $85 \%$ to $100 \%$ in larger case series. ${ }^{4-7}$ When resecting cavernous malformations in any location, success is measured first by the complete removal of the symptomatic lesion and second by the absence of new permanent neurological deficits. Curative resection at the time of index surgery rids the patient of the offending lesion and eliminates the risk of future deterioration caused by rehemorrhage, at least in the sporadic form of the disease. Surgical success is common with most cavernous malformations in the cerebral and cerebellar hemispheres. Still, resection of cavernous malformations in the brainstem is challenging. The proximity of BSCMs to the descending and ascending tracts and $\mathrm{CN}$ nuclei make it costly to transgress any normal tissue in accessing the lesion or disrupting any normal tissue adjacent to the lesion in the separation plane. This interplay between tissue sensitivity and extreme eloquence makes it difficult, if not impossible, to avoid leaving a remnant on occasion. The largest institutional surgical case series by a single neurosurgeon reported a rate of residual or recurrent BSCM of $11 \%$ ( 29 of 260 patients). ${ }^{4}$

The natural history and management of residual or recurrent BSCMs are poorly described in the literature. ${ }^{4,8-10}$ Current findings suggest that completely resected BSCMs do not recur de novo in the same location, which implies that some residual BSCM or remnants are likely present but poorly visualized immediately after resection. ${ }^{4,9}$ BSCM recurrence is associated with many difficulties. For the patient, it is a clinical setback that can generate frustration, disappointment, and additional morbidity. For the neurosurgeon, recurrence can rattle surgical self-confidence, cause embarrassment, and diminish the trust of patients and colleagues. Symptomatic recurrences require operative intervention, which may increase morbidity, lengthen recovery, and add to overall hospitalization and rehabilitation costs. Although rare, this problem deserves special attention. We therefore reviewed an approximately 20-year experience with BSCM among patients with recurrent lesions following primary microsurgical resection.

\section{Methods \\ Study Population}

This study was approved by the Committees on Human Research at the University of California, San Francisco, and the Barrow Neurological Institute. The prospectively maintained database of the senior author (M.T.L.) was queried to identify 802 patients who underwent microsurgical resection of a cavernous malformation in the brain between October 1, 1997, and November 30, 2018. From this cohort, a retrospective chart review was conducted for 213 patients who underwent microsurgical resection of BSCMs during this period. Patients with recurrent BSCM after primary resection who required a second surgery were included in the study. Patients were included only if the primary and secondary surgeries were performed by the senior author; 9 patients with symptomatic hemorrhage from recurrent BSCM after resection performed by another neurosurgeon were excluded. Two patients with recurrent BSCM after resection performed by another neurosurgeon were included because each had a second recurrence after surgery that was treated by the senior author (3 BSCM operations in total were performed for each patient). Lesions located entirely within the basal ganglia or thalamus were excluded as well.

\section{Data Collection}

All data were retrospectively collected from clinical assessments (at admission, during the immediate postoperative period, and at last follow-up), radiographic imaging, operative notes, intraoperative photographs and videos, postoperative imaging, and final pathology reports. The senior author dictated all operative notes. An independent neuroradiologist reviewed all neuroimaging data. All patients included in the data set had pathologically proven cavernous malformations. Operative notes and radiographic images were used to determine the presence of developmental venous anomalies. The modified Rankin Scale (mRS) score was utilized to assess and quantify patient outcomes. ${ }^{11}$ The outcome was considered favorable if the patient had an mRS score $\leq 2$ at the time of the final clinic evaluation; an mRS score $>2$ was considered an unfavorable score. The timing of rehemorrhage was defined from the date of surgical intervention to the date of clinical rehemorrhage or radiographic evidence of hemorrhage or finding of a residual lesion on postoperative imaging, after radiographic evidence of rehemorrhage.

\section{Statistical Analysis}

Summary statistics were reported using the median and IQR due to small sample bias. Descriptive statistical analysis was conducted using the Wilcoxon rank-sum test and Fisher's exact test for continuous and categorical variables, respectively, given small cell counts. Statistical tests were considered significant if $\mathrm{p}<0.05$. All statistical analyses were performed using Stata Intercooled version 14 (StataCorp).

\section{Results \\ Patient Characteristics and Presentation}

A total of 14 of 213 patients (6.6\%) underwent a second resection for recurrent BSCM after primary resection (Table 1). The median age of patients who underwent a second resection for recurrent BSCM was 32 years (IQR $32-41$ years), with a sex preponderance toward female patients $(\mathrm{n}=13)$.

Nine patients were clinically symptomatic at the time of recurrent hemorrhage and presented with worsening neurological deficits. Five patients had asymptomatic recurrences detected on surveillance MRI at follow-up imaging (cases 1, 2, 3, 10, and 13). Two patients (cases 2 and 3) had suspicious immediate postoperative imaging and underwent multiple postoperative scanning sessions until the surgical remnant became evident during hospitalization. Three patients (cases 1, 10, and 13) did not have any neurological deterioration or remnants found on MRI immediately after surgery, but hemorrhagic blood products (cases 1 and 10) or a remnant (case 13) was ultimately identified. The median time from surgical intervention to a remnant or asymptomatic rehemorrhage without neu- 


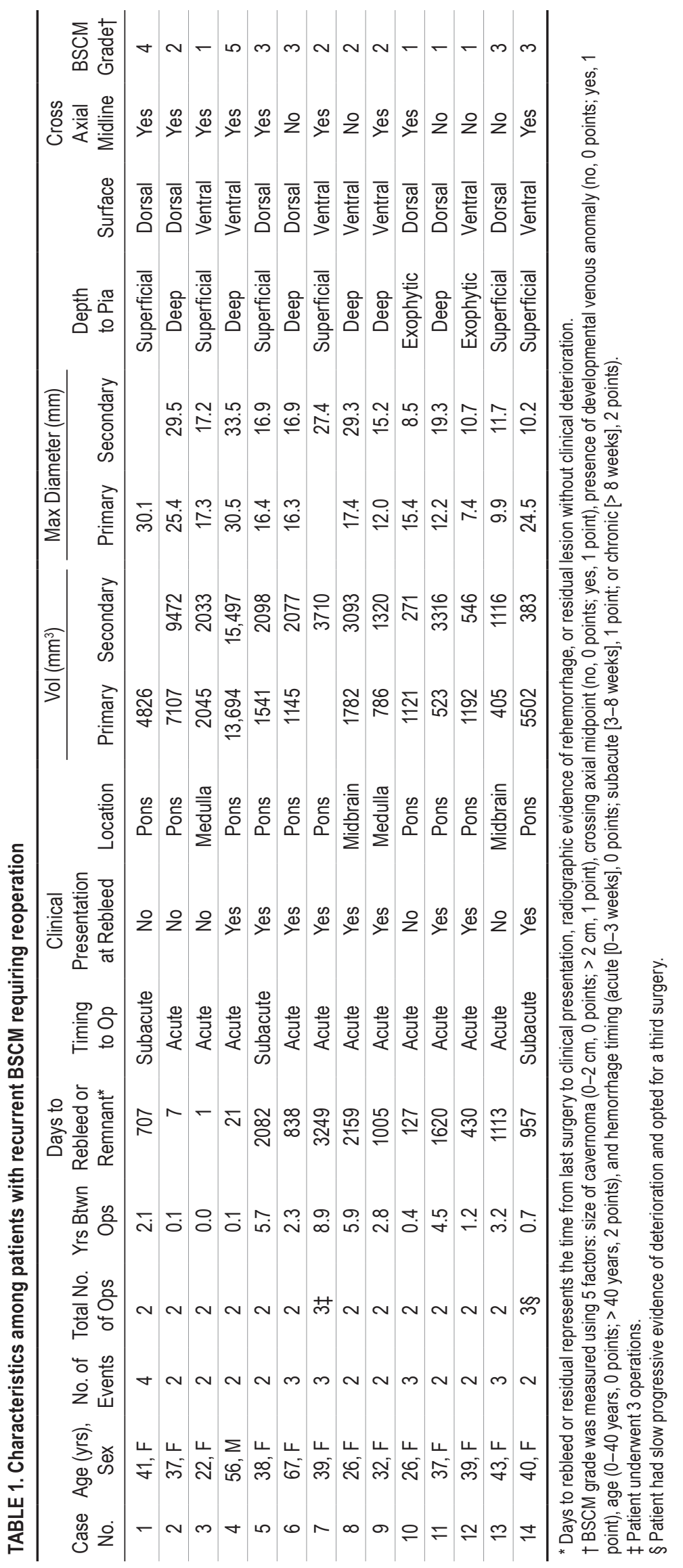


rological deterioration was 127 days (IQR 4-906 days). Overall, there were 34 hemorrhagic events among these 14 patients over 576 patient-years, resulting in a retrospective recurrent hemorrhage rate of $5.9 \%$, or 5.9 events per patient-year. The median number of discrete hemorrhagic events was 2 (IQR 1-3 events). The median time from the last surgery to rehemorrhage was 1005 days (IQR 6342211 days) among those without evidence of remnant on postoperative neuroimaging.

\section{BSCM Characteristics}

Three patients had multiple cerebral cavernomas, and 11 had single sporadic BSCMs, with 23 total lesions among the 14 patients. Recurrent BSCM was located in the pons in 10 cases, in the midbrain in 2 cases, and in the medulla in 2 cases. Seven lesions had ventral locations. The median maximum diameter was $16.9 \mathrm{~mm}$ (IQR 11.7$27.4 \mathrm{~mm}$ ). The median distance to the pial surface was $0.25 \mathrm{~mm}$ (IQR 0-2.0 mm). Two recurrent BSCMs were exophytic, 6 abutted the pial surface, and 6 were deep. Eight patients had a concurrent developmental venous anomaly that drained the index BSCM. BSCM grades are shown in Table 1.

\section{Surgical Results}

The extended retrosigmoid $(\mathrm{n}=6)$ and suboccipitaltransventricular $(n=5)$ surgical approaches were the most common. Two far-lateral approaches, 1 orbitozygomaticpterional approach, 1 lateral supracerebellar-infratentorial approach, and 1 translabyrinthine approach were also performed (Table 2).

Eleven of the 14 patients underwent primary surgical treatment in an acute time frame (less than 3 weeks), and 3 patients underwent primary surgical treatment in a subacute time frame (3-8 weeks) (Table 1). Two patients underwent a second operation for recurrence (third surgery overall). The first patient (case 7) experienced rebleeding 8.5 years after the second operation. The other patient (case 14) underwent resection 8.5 months after her second resection when a residual lesion was found on her 6-month surveillance MRI. In these 2 cases of pontine BSCMs, the initial incomplete resection was performed by another neurosurgeon, and the exposure in both cases was deemed by the senior author to be inadequate. In one patient (case 7), the prior retrosigmoid approach was expanded to a translabyrinthine approach. In the other patient (case 14), the prior "mini" retrosigmoid craniotomy was expanded to an extended retrosigmoid craniotomy.

A review of intraoperative photographs and videos determined that a blind spot in the operative corridor was the most common cause of residual BSCM, which was found in 9 patients (Figs. 1 and 2). An anatomical obstacle hid the remnant in 2 patients (a developmental venous anomaly in 1 patient and $\mathrm{CN}$ IX and $\mathrm{X}$ in 1 patient). In 2 patients, the BSCM was in clear view, but the tissue at the depth of the cavity was misinterpreted as normal brainstem rather than a remnant of the lesion (Fig. 3). Recurrent BSCM was found at reoperation at this exact location in these 2 patients. In one patient, the recurrence was felt to be a satellite lesion immediately adjacent to the main BSCM (Fig. 4).

\section{Patient Outcomes}

The mean hospitalization time was 8 days (IQR 6-11 days), and the median follow-up time was 15 months (IQR 6-39 months). There were no intraoperative complications or deaths among the patient cohort. Four patients developed medical complications or required additional procedures: ventriculoperitoneal shunt (case 2), gastrostomy tube placement (case 4), pseudomeningocele (case 10), and chemical meningitis (case 13).

Overall, patients improved neurologically after treatment. All patients initially presented with at least one focal neurological deficit (1 patient presented with obtundation, 7 patients had motor deficits, 11 had at least one cranial nerve deficit, 7 had sensory dysfunction, and 3 had cerebellar dysfunction or ataxia). In the immediate postoperative period, 2 patients had worsening cranial neuropathies, and 1 had new mild hemiparesis $(4+/ 5$, case 14$)$ after the third resection; otherwise, all patients remained at neurological baseline. At the time of the last clinical evaluation, 2 patients had motor deficits, 5 patients had sensory dysfunction, and 1 patient had cerebellar dysfunction. Four patients had made a significant recovery in their cranial nerve deficits. Overall, 13 patients had at least one $\mathrm{CN}$ deficit at the time of the last follow-up. Twelve patients had mRS scores that were unchanged or improved at the last clinical evaluation compared with $\mathrm{mRS}$ scores at admission, and 2 patients had worse mRS scores (Table 2).

\section{Neurosurgeon Experience}

Although the overall recurrence rate was $6.6 \%$, recurrence was more common among patients who were operated on in the first half of the series than those operated on in the second half. Overall, 9 recurrences were observed among the first 106 patients $(8.5 \%)$, and 5 recurrences were observed among the last 107 patients (4.7\%), which suggests that increased surgeon experience may be associated with lower recurrence rates (Fig. 5).

\section{Discussion}

In this consecutive series of patients with BSCMs treated microsurgically over 20 years, the rate of recurrence requiring reoperation was $6.6 \%$ (14 of 213 patients), with all but 2 of these patients presenting with acute clinical deterioration from new hemorrhage. Patients experienced rebleeding an average of 2.5 years after their primary resection, which is comparable to the timing reported in other large institutional case series ${ }^{12}$ and which demonstrates the difficulty of detecting remnants. With our management protocol, postoperative MRI is performed within 48 hours after resection to identify obvious remnants, and patients with positive imaging findings are returned to the operating room to complete the resection. There was no immediate return to the operating room in this experience. However, 2 patients (cases 2 and 3) remained hospitalized due to inconclusive imaging findings and ultimately underwent resection. If no residual BSCM is found in the immediate postoperative period, a follow-up MRI is done at 6 months to evaluate the cavity after the blood has been resorbed and the tissues have healed. Surveillance imaging is recommended again at 5 years. There were an 


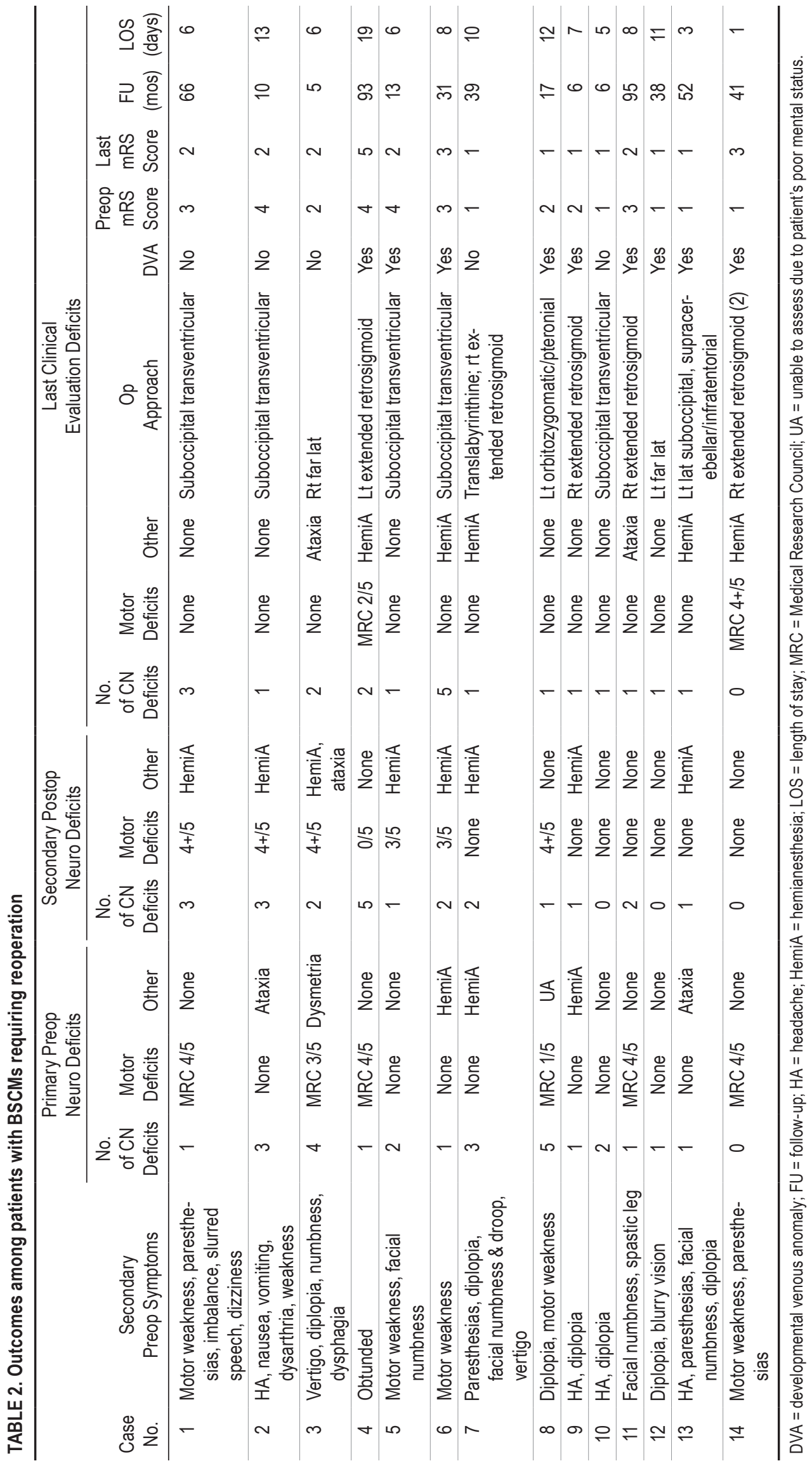



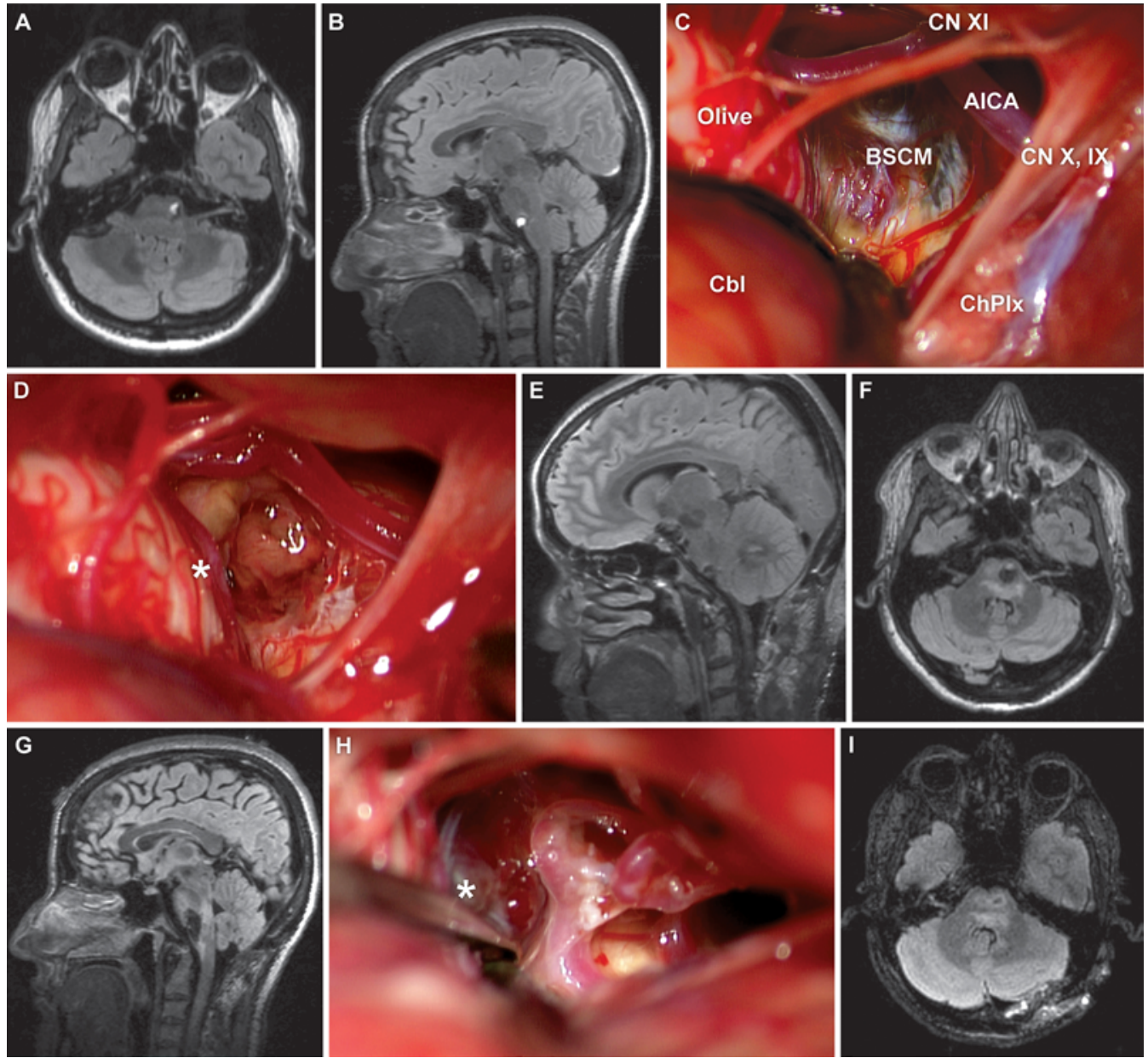

FIG. 1. Case 12. A residual pontine cavernous malformation hid in the inferior blind spot of a left far-lateral/transpontomedullary sulcus approach, behind the olivary nucleus. This 39-year-old woman presented with diplopia and blurred vision. A and B: Axial (A) and sagittal (B) T2-weighted FLAIR MR images demonstrating the lesion. C: A far-lateral craniotomy exposed the lesion in the

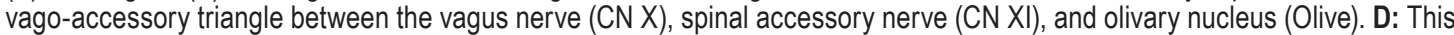
exophytic lesion was resected through a transpontomedullary sulcus approach, but the residual lesion remained inferiorly behind the olivary nucleus (asterisk). E: Sagittal T2-weighted FLAIR MR image showing no residual lesion postoperatively, but the patient presented 14 months later with symptomatic rehemorrhage. F and G: Axial (F) and sagittal (G) T2-weighted FLAIR MR images showing evidence of hemorrhage. $\mathrm{H}$ : At reoperation through the same left far-lateral craniotomy, the cavernous malformation (asterisk) was found in the previous resection cavity inferiorly, behind the olivary nucleus. I: Postoperative axial T2-weighted FLAIR MR image confirming complete removal of the lesion. The patient recovered with no new neurological deficits. AICA = anterior inferior cerebellar artery; $\mathrm{Cbl}=$ cerebellum; $\mathrm{ChPIx}=$ choroid plexus; $\mathrm{CN}$ IX = glossopharyngeal nerve. Panels $\mathrm{C}, \mathrm{D}$, and $\mathrm{H}$ : copyright Barrow Neurological Institute, Phoenix, Arizona. Published with permission.

additional 3 returns (cases 1, 10, and 13) to the operating room in this experience after positive surveillance imaging. This vigilant protocol failed to identify residual or recurrent lesions in 9 of the 14 patients, demonstrating the difficulty of detecting tiny remnants in the perioperative period and also suggesting that new hemorrhage may be needed to bring them to detection. Tissue edema, hemostatic agents, and pooled blood in the resection cavity can hide a remnant, which is why we pursue hemostasis meticulously with bipolar cautery, avoid the use of oxidized cellulose products inside the cavity or to line its walls, and fill the resection bed with irrigant before closing. If surveillance imaging at 6 months or later identifies a remnant lesion, the reappearance often results from bleeding activity, as in both of our cases. Hemorrhagic remnants are associated with a significant risk of additional rehemorrhage and morbidity, and therefore surgical intervention is recommended even when the patient is clinically asymptomatic. Routine surveillance should continue with MRI 5 years after resection of a recurrent BSCM to ensure that a complete resection has been achieved. Conservative management of asymptomatic remnants with hemorrhagic activity is not usually recommended but may be appropriate when the reoperative risk is high or the patient prefers observation.

A 93.4\% complete resection rate is consistent with other reports in the literature, but some of the 199 patients who were not operated on whose BSCM was deemed to have been cured may have asymptomatic recurrences that have not yet bled or been diagnosed. Therefore, the $6.6 \%$ 

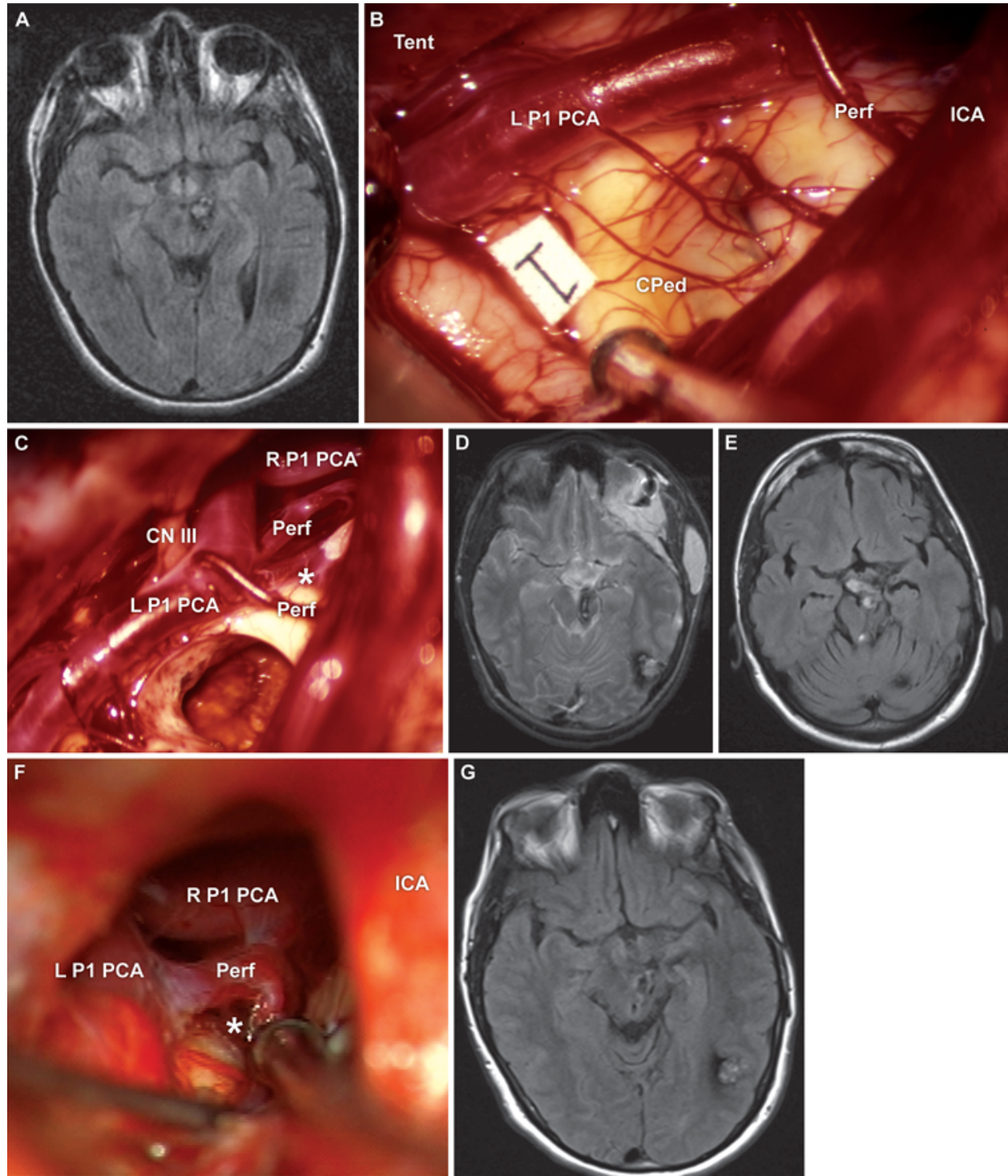

FIG. 2. Case 8. A residual midbrain cavernous malformation hid in the medial blind spot under the cerebral peduncle after a left orbitozygomatic-pterional/transsylvian approach. This 26-year-old woman presented with diplopia and hemiparesis. A: Axial T2-weighted FLAIR MR image demonstrating the lesion. B: Through a left orbitozygomatic-pterional craniotomy and transsylvian approach, the cerebral peduncle (CPed) was exposed and mapped to identify the descending motor tract (1). C: The lesion was resected medial to the motor tract through the hemosiderin stain on the CPed. The lesion was accessed between the thalamoperforators (Perf), but residual lesion remained medially (asterisk). D: Axial T2-weighted FLAIR MR image showing no residual lesion postoperatively, but the patient presented 6 years later with symptomatic rehemorrhage. E: Axial T1-weighted MR image showing evidence of hemorrhage. F: At reoperation through the same left orbitozygomatic-pterional craniotomy/transsylvian approach, the recurrent cavernous malformation was found medial to the previous resection cavity, underneath a more medial thalamoperforator trunk (asterisk). G: Axial T2-weighted MR image confirming complete removal of the lesion. The patient recovered with no new neurological deficits. $\mathrm{CN}$ III = oculomotor nerve; ICA = internal carotid artery; PCA = posterior cerebral artery; Tent = tentorium.

Panels B, C, and F: copyright Barrow Neurological Institute, Phoenix, Arizona. Published with permission.

recurrence rate in our study may be an underestimate. In addition, patients with recurrent BSCM who opted to forego repeat surgery are difficult to identify retrospectively. Although we do not know of any such patients in this series, they would not have been included in the study. The bleeding behavior of recurrent BSCM in this experience, namely, the 5.9\% annual hemorrhage risk, is consistent with that reported for BSCMs not operated on and appears to be associated with a similar clinical course.

\section{Surgical Blind Spots}

Blind spots, or areas in the surgical corridor with a compromised or inadequate view, were identified as the most common cause of incomplete BSCM resection in this experience. The inherent depth of lesions in the brainstem and the length of the corridor make blind spots inevitable. The surgical corridor comprises 1) the craniotomy, 2) a subarachnoid or ventricular segment, 3) a parenchymal segment, and 4) an intralesional segment (Fig. 6). Each 

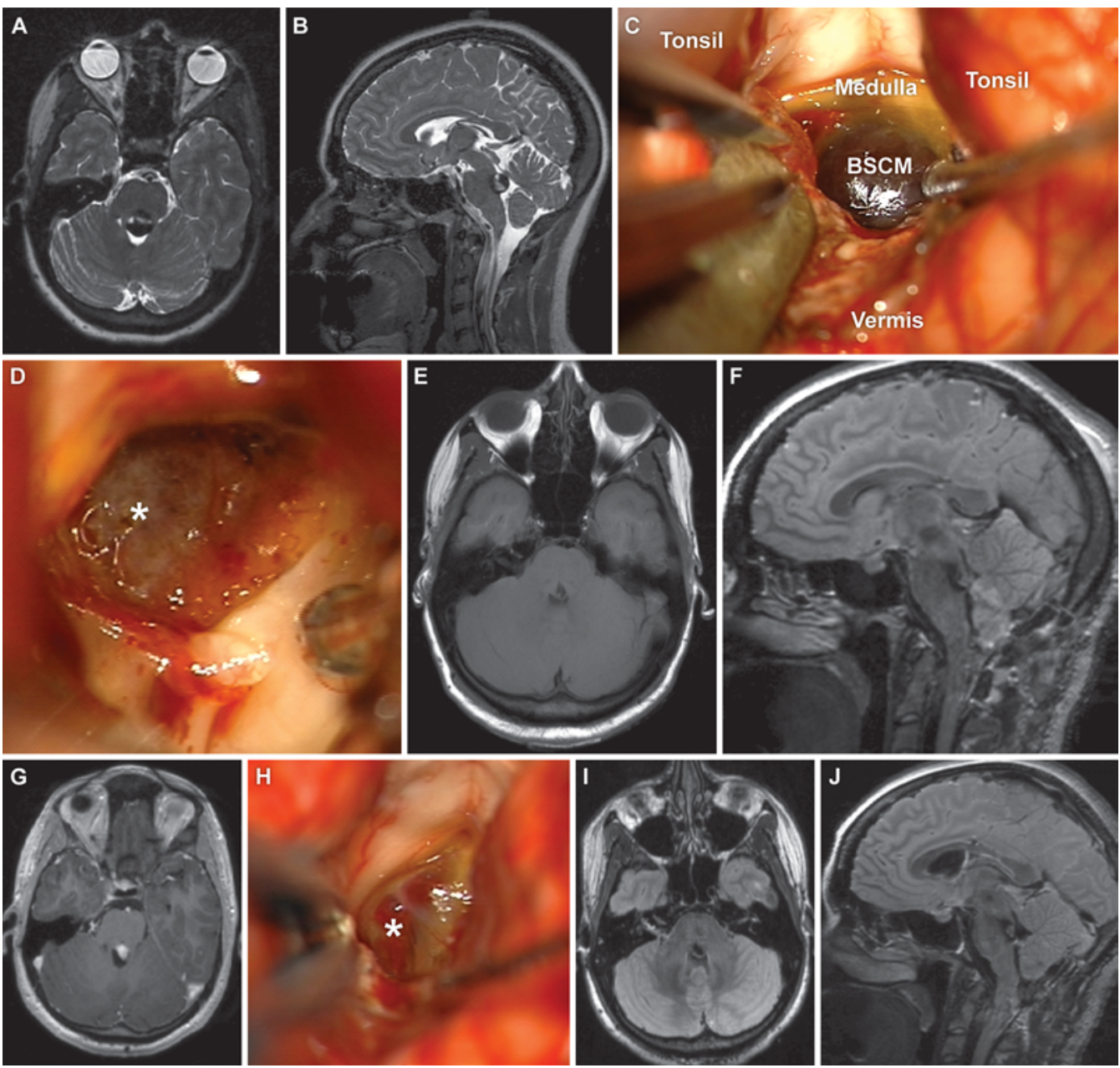

FIG. 3. Case 10. Recurrence resulted from misinterpretation of the deep margin of the cavernous malformation. This 26 -year-old woman presented with headache and diplopia. A and B: Axial (A) and sagittal (B) T2-weighted MR images demonstrating the lesion. C: A suboccipital craniotomy and transventricular approach easily exposed the lesion on the floor of the fourth ventricle. D: After what was thought to be a complete resection, the deep wall of the resection cavity was interpreted as gliotic pons (asterisk). E and F: Postoperative axial T1-weighted (E) and sagittal T2-weighted (F) FLAIR MR images do not appear to suggest residual BSCM, but when the patient presented for routine surveillance imaging without evidence of neurological deterioration, there was radiographic evidence of rehemorrhage. G: Axial T1-weighted MR image showing evidence of hemorrhage. H: At reoperation through the same suboccipital craniotomy, recurrent cavernous malformation was found at the depths of the previous resection cavity (asterisk). Deeper dissection into the cavity exposed the residual lesion, and it was removed completely. I and J: Axial (I) and sagittal (J) T2-weighted FLAIR MR images demonstrating complete removal of the lesion. The patient recovered with no new neurological deficits. Panels C, D, and H: copyright Barrow Neurological Institute, Phoenix, Arizona. Published with permission.

component adds length and limits visualization. For example, the trans-middle cerebellar peduncle approach to a pontine BSCM has a corridor made up of the retrosigmoid craniotomy, subarachnoid dissection into the cerebellopontine angle, a tunnel through the middle cerebellar peduncle, and entrance into the lesion. The view of the lesion through this 6-cm corridor can be obscured by the edges of the craniotomy, a prominent flocculus, the collapsing white matter of the middle cerebellar peduncle, or the corners at the interface between the middle cerebellar peduncle and the lesion laterally. Corridors to BSCMs have small openings, long lengths, and limited viewing angles. This situation is the opposite of the keyhole concept in which a small opening plus a wide range of views still enable a good survey of the lesion. With BSCMs, the keyhole is displaced to the end of an already long corridor without that range of viewing angles, which decreases visualization and requires that the lesion be mobilized into the viewing axis. The blind spots created by this geometry force possible blind dissection and hide the pathology. One must conceptualize the blind spots and deliberately inspect these spaces at the end of the resection, even after the resection seems complete.

This difficult geometry is compounded by darkness, swollen brain tissue, and poor approach selection. Lighted instruments (bipolar forceps and suction) in long corridors are invaluable. Brain edema can narrow the corridor and make the walls more susceptible to injury with manipulation and retraction. Delaying surgery for at least 2 weeks after a hemorrhage allows time for edema to resolve and 

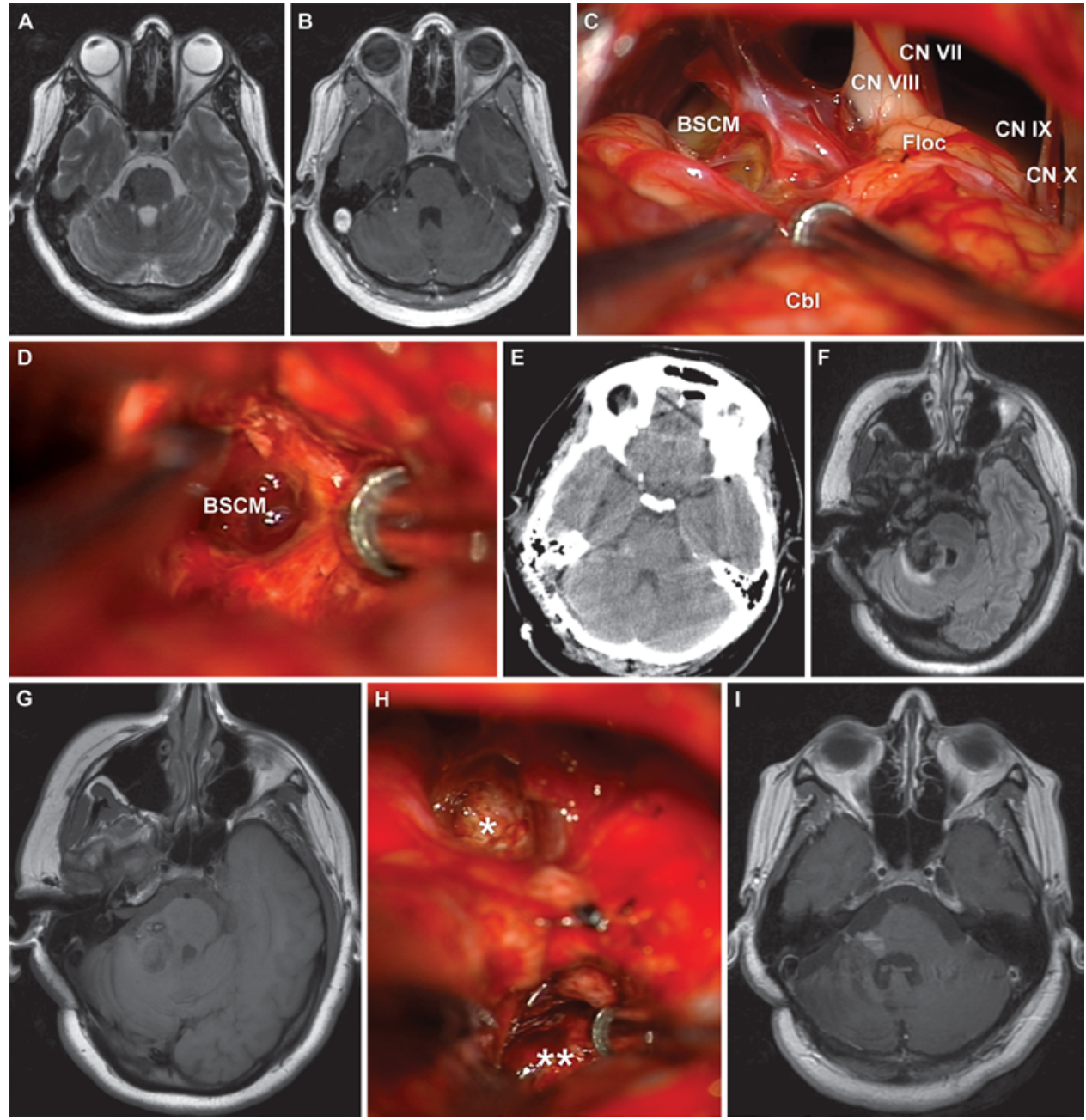

FIG. 4. Case 11. This 37-year-old woman presented with right facial numbness. A and B: Axial T2-weighted (A) and T1-weighted Gd-enhanced (B) MR images demonstrating a cavernous malformation in the right lateral pons at the level of the trigeminal root entry zone. Note the satellite cavernous malformation in the middle cerebellar peduncle, lateral to the pontine lesion. C: An extended retrosigmoid craniotomy and dissection into the cerebellopontine angle exposed the greenish hemosiderin stain on the lateral pontine surface, superior to the facial (CN VII) and vestibular (CN VIII) nerves. D: The lesion was identified in the lateral pons and removed. E: Postoperative CT scan showing no findings suggestive of a residual lesion. MRI also lacked findings suggestive of a residual lesion (not shown). F and G: Axial T2-weighted FLAIR (F) and T1-weighted (G) MR images showing new hemorrhage at the site of the prior surgery 4.5 years later (at 37 years of age). The hematoma was contiguous with the unresected satellite lesions seen previously in the middle cerebellar peduncle. $\mathrm{H}$ : At reoperation through an extended retrosigmoid craniotomy, the satellite lesion was resected through a transfloccular/trans-middle cerebellar peduncle approach (double asterisks), and a small recurrence was resected at the previous resection cavity in the lateral pons (asterisk). I: Postoperative axial T1-weighted MR image confirming complete resection. The patient recovered with no new neurological deficits. $\mathrm{Cbl}=$ cerebellum; $\mathrm{CN} \mathrm{VII}=$ facial nerve; $\mathrm{CN}$ VIII = vestibulocochlear nerve; $\mathrm{CN} I X=$ glossopharyngeal nerve; $\mathrm{CN} X=$ vagus nerve; Floc = flocculus. Panels $\mathrm{C}, \mathrm{D}$, and $\mathrm{H}$ : copyright Barrow Neurological Institute, Phoenix, Arizona. Published with permission.

associated hematoma to liquefy, both of which make it easier to dissect the BSCM separation planes. Dynamic retraction is important within deep corridors because it shifts the tissues, viewing axis, and illumination slightly with each move to gain a glimpse into blind spots. Fixed retraction is rarely helpful in seeing within the resection cavity and often obstructs the dissection maneuvers.

\section{Right-Angle Method}

The "2-point method" has proven to be a useful guide to select the correct approach to BSCMs. ${ }^{13}$ The first point is placed where the BSCM comes to a pial or ependymal surface. The second point is placed at the center of the lesion, and the line connecting these 2 points defines the lesion's axis. The 2-point method is a heuristic that matches the lesion's axis with the surgical axis, which is the trajectory of the operative corridor. When these axes align, the lesion is well visualized within the resection cavity; when these axes do not align, visualization is impaired.

One way to conceptualize blind spots is with another method: the "right-angle method." The surgical axis is defined by 2 points: one at the superficial entrance into the operative corridor (corridor entry, point 1) and one at the 


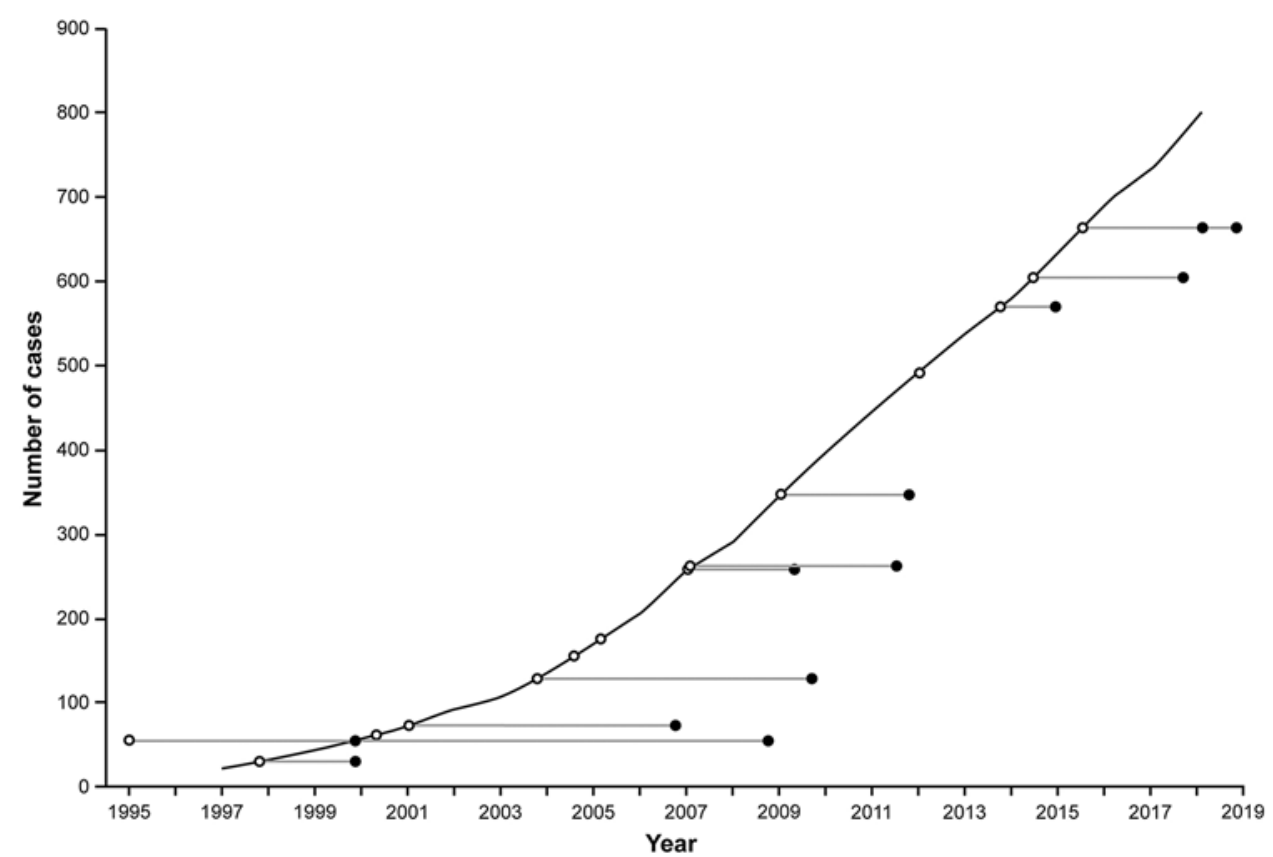

FIG. 5. Effect of neurosurgeon experience on the incidence of residual or recurrent BSCMs. The cumulative case volume of all cerebral cavernous malformations resected by the senior author during an approximately 20 -year period is plotted. Patients with residual or recurrent BSCM are superimposed chronologically as horizontal lines, with the line beginning at the time of the first operation and ending at the time of the last operation. Initial operations are indicated with a white dot, and operations to treat residual or recurrent BSCM are indicated with a black dot. If the second operation occurred less than 6 months after the initial operation, both operations are indicated with a single white dot. Two patients underwent 3 operations; for one of these patients, the initial operation was performed by another surgeon in 1995.

entry into the BSCM (front wall, point 2; Fig. 7). The surgical axis is drawn by connecting these points and extending this line through the lesion to its back wall (point 3). With the right-angle method, a perpendicular line is drawn from the surgical axis to the point on the lesion's capsule farthest from the axis. Lobulations and compartments often make these lesions irregular and asymmetrical, rather than simple spheres. Perpendicular lines drawn on axial, sagittal, and coronal MR images identify multiple potential blind spots, and any perpendicular line exceeding 1 $\mathrm{cm}$ in length identifies a potential blind spot. The surgical axis approximates the line of sight into the resection bed, which makes spots in the near corners more difficult to see than those in the far corners. Sight down long corridors cannot make right turns, which transforms ledges of eloquent tissue in the near field into blind spots. The rightangle method helps anticipate the blind spots, which drives meticulous inspection for residual BSCM during surgery (Fig. 7).

\section{The Fine Line}

After blind spots, misinterpretation was the next most common cause of residual or recurrent lesions. Every resection requires the painstaking evaluation of the plane that separates the cavernous malformation from the brainstem itself. In other words, overcoming blind spots and obtaining the proper views brings the greater challenge of dissecting at the border between the lesion and parenchyma, between morbidity and cure. Direct visualization and cau- tious dissection define these differences. Sometimes color, contour, and texture make this distinction obvious, with mulberry-like lesions embedded in hemosiderin-stained parenchyma or a thick capsule that peels away cleanly from the brainstem. Other times, hemorrhagic parenchyma blends indistinctly with the pathology, as in 2 cases in which the remnant was not in a blind spot but left behind in plain sight. The brainstem's supreme eloquence can make neurosurgeons cautious. Conservatism keeps operations safe and patients intact, whereas probing deeper and pushing further can be devastating. At these moments of uncertain interpretation, completeness and morbidity pull at each other like a tug-of-war in one's mind. This perception is the fine line that separates complete resection from recurrence and good outcomes from new deficits.

The capsule is key to safe and complete lesionectomy. A small opening into the capsule is necessary to drain the liquefied hematoma and reduce the lesion's size. Caverns are opened and interconnected internally to explore the different portions of the lesion. Capsular preservation keeps the lesion whole and protects the brainstem from the neurosurgeon's maneuvers. Gradually, the capsule pulls away from the parenchyma. The dissection then switches from intracapsular to extracapsular, freeing adhesions circumferentially and collapsing the capsule inward to find remaining points of adhesion. Ideally, circumferential dissection liberates the BSCM with its capsule intact, except for the initial entry site. Sharp dissection is performed with round knives of varying sizes and angles and rarely with microscissors. Delicate traction with a grasping in- 


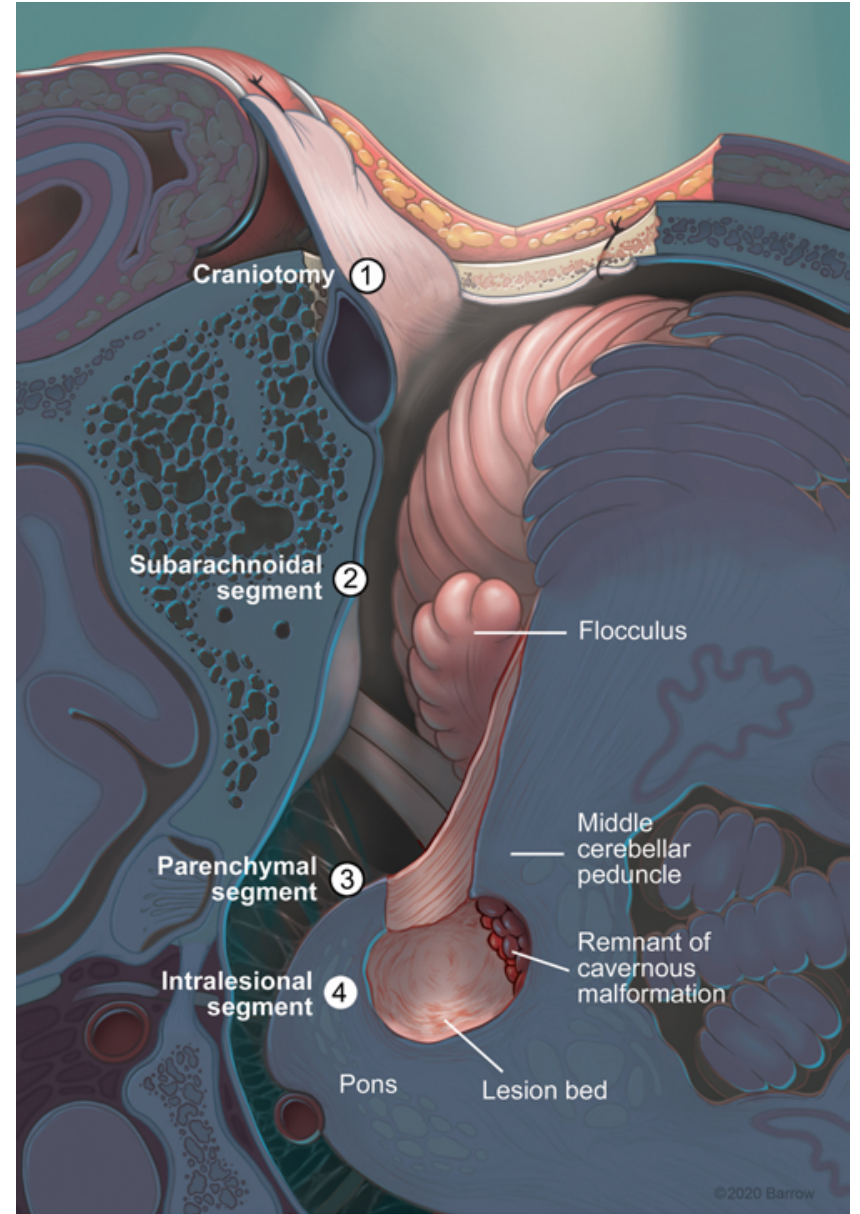

FIG. 6. Illustration of the anatomy of the blind spot. The inherent depth of a BSCM and the length of the corridor required to reach it make blind spots inevitable. The surgical corridor comprises 1) the craniotomy; 2) a subarachnoid, cisternal, or ventricular segment; 3) a parenchymal segment; and 4) an intralesional segment. With a BSCM, the small keyhole opening into the lesion is displaced to the end of a very long corridor that limits the viewing angles, decreases visualization, and creates blind spots in which a remnant can hide. Copyright Barrow Neurological Institute, Phoenix, Arizona. Published with permission.

strument and countertraction with the suction finally deliver the lesion.

Neurophysiological monitoring identifies areas of highest eloquence, like the facial colliculus in the fourth ventricular floor or the pyramidal tract in the cerebral peduncle of the midbrain. Neuromonitoring can also warn the neurosurgeon when the dissection is too rough or strays into monitored tracts and nerves. Although these alarms help redirect or modulate the dissection, they can also derail the neurosurgeon. A sudden signal change elicits worry and hesitation, often at the end of a resection, when the borders of the lesion have been reached. Too much caution at the finish line can easily lead to leaving a BSCM remnant. Small diminutions of signals should not deter the pursuit of curative resection because they often translate to transient deficits postoperatively. The information must be processed without impeding progress to complete resection. Neuromonitoring has become a standard of care for

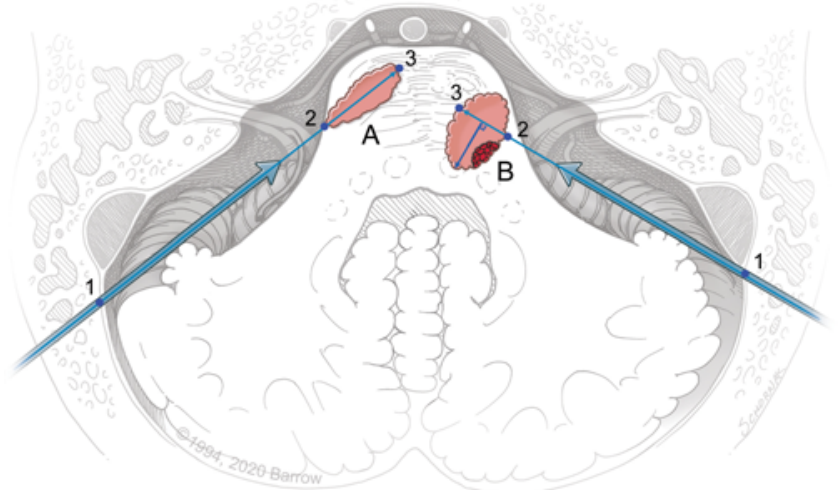

FIG. 7. Illustration of the right-angle method. The surgical axis is defined by 2 points: one at the superficial entrance into the operative corridor (corridor entry, point 1) and one at the entry into the BSCM (front wall, point 2). The surgical axis is drawn by connecting these points and extending this line through the lesion to its back wall (point 3). The rightangle method draws a perpendicular line from the surgical axis to the point on the lesion's capsule farthest from the axis. Any perpendicular line exceeding $1 \mathrm{~cm}$ in length is identified as a potential blind spot. This illustration shows two pontine cavernous malformations, each exposed through an extended retrosigmoid/trans-middle cerebellar peduncle approach. The long axis of lesion A aligns with the surgical axis, and the right-angle method identifies no blind spot. In contrast, the long axis of lesion B does not align with the surgical axis, and the right-angle method identifies a posterolateral blind spot hiding a remnant (red) of the cavernous malformation. Copyright Barrow Neurological Institute, Phoenix, Arizona. Published with permission.

this operation but requires a steeled mindset to press onward when neurophysiological signals change, representing another fine line to be managed.

The fine lines between complete resection and recurrence, between safe and harmful resection, and between heeding and ignoring neuromonitoring make this operation one of the most challenging in neurosurgery. It lacks the drama of intraoperative rupture or the elegance of deep bypasses, but it demands circumspection at every step, from the approach selection to the exposure to each maneuver during the resection. It demands constant calculation regarding how hard to pull, how deep to probe, and how thorough to search. While these other challenging operations are in subarachnoid spaces or deep cisterns, the resection of a cavernous malformation is within the brainstem, which raises the stakes to the highest level. Even extensive neurosurgeon experience does not seem to eliminate the problem of incomplete resection. Residual lesions were observed less frequently in the second half of this experience, which reflects the maturation of technique, comfort working within the brainstem, awareness of blind spots, and a feel for separation planes. However, recurrences were still observed late in this surgical experience of more than 800 cerebral cavernous malformations. One of the greatest benefits of experience is that it lessens the uncomfortable dissonance that comes from walking these fine lines. The enduring problem of residual BSCM, despite extensive experience, highlights the need for a neurosurgeon to constantly modulate between an aggressive approach, associated with undue operative morbidity, and a conservative approach, associated with high rates of re- 
currence. Recurrent BSCM occurs in 5\%-10\% of patients, and this possibility ensures that we constantly work toward an acceptable balance of safety and completeness.

\section{Conclusions}

In this large series of patients with BSCM treated microsurgically over more than 20 years, the recurrence rate in patients requiring reoperation was $6.6 \%$ (14 of 213 patients), with all but 2 presenting with acute clinical deterioration from a new hemorrhage. Detecting remnants is difficult during the immediate postoperative period and even 6 months after the operation, when the resection bed has healed. The 5.9\% annual hemorrhage risk of recurrent $\mathrm{BSCM}$ in this experience is consistent with that reported for unoperated BSCMs. Blind spots were the most common cause of incomplete BSCM resection in this experience. The right-angle method helps to anticipate blind spots and meticulously inspect the resection cavity for residual BSCM during surgery. The percentage of patients with BSCM who experience recurrence is low (5\%-10\%), which ensures that we constantly work toward an acceptable balance of safety and completeness.

\section{Acknowledgments}

Dr. Roxanna Garcia served as the StrokeNet research fellow from 2018 to 2019 and is a Fogarty Global Health Trainee from 2019 to 2020, Department of Neurological Surgery, Northwestern University, Feinberg School of Medicine. Research reported in this publication was supported under the StrokeNet award number U24 NS107233-01 and the Fogarty International Center and National Institute of Mental Health, of the National Institutes of Health, under award number D43 TW010543. The content is solely the responsibility of the authors and does not necessarily represent the official views of the National Institutes of Health.

We thank the staff of Neuroscience Publications at Barrow Neurological Institute for assistance with manuscript and illustration preparation.

\section{References}

1. Gross BA, Batjer HH, Awad IA, Bendok BR. Brainstem cavernous malformations. Neurosurgery. 2009;64(5):E805E818.

2. Gross BA, Batjer HH, Awad IA, et al. Brainstem cavernous malformations: 1390 surgical cases from the literature. World Neurosurg. 2013;80(1-2):89-93.

3. Gross BA, Du R. Hemorrhage from cerebral cavernous malformations: a systematic pooled analysis. J Neurosurg. 2017; 126(4):1079-1087.

4. Abla AA, Lekovic GP, Turner JD, et al. Advances in the treatment and outcome of brainstem cavernous malformation surgery: a single-center case series of 300 surgically treated patients. Neurosurgery. 2011;68(2):403-415.
5. Garcia RM, Ivan ME, Lawton MT. Brainstem cavernous malformations: surgical results in 104 patients and a proposed grading system to predict neurological outcomes. Neurosurgery. 2015;76(3):265-278.

6. Wang CC, Liu A, Zhang JT, et al. Surgical management of brain-stem cavernous malformations: report of 137 cases. Surg Neurol. 2003;59(6):444-454.

7. Pandey P, Westbroek EM, Gooderham PA, Steinberg GK. Cavernous malformation of brainstem, thalamus, and basal ganglia: a series of 176 patients. Neurosurgery. 2013;72(4): 573-589.

8. Cenzato M, Stefini R, Ambrosi C, Giovanelli M. Post-operative remnants of brainstem cavernomas: incidence, risk factors and management. Acta Neurochir (Wien). 2008;150(9): 879-887.

9. Abla AA, Lekovic GP, Garrett M, et al. Cavernous malformations of the brainstem presenting in childhood: surgical experience in 40 patients. Neurosurgery. 2010;67(6):1589-1599.

10. Bertalanffy H, Gilsbach JM, Eggert HR, Seeger W. Microsurgery of deep-seated cavernous angiomas: report of 26 cases. Acta Neurochir (Wien). 1991;108(3-4):91-99.

11. van Swieten JC, Koudstaal PJ, Visser MC, et al. Interobserver agreement for the assessment of handicap in stroke patients. Stroke. 1988;19(5):604-607.

12. Steinberg GK, Chang SD, Gewirtz RJ, Lopez JR. Microsurgical resection of brainstem, thalamic, and basal ganglia angiographically occult vascular malformations. Neurosurgery. 2000;46(2):260-271.

13. Brown AP, Thompson BG, Spetzler RF. The two-point method: evaluating brain stem lesions. BNI Q. 1996;12(1):20-24.

\section{Disclosures}

The authors report no conflict of interest concerning the materials or methods used in this study or the findings specified in this paper.

\section{Author Contributions}

Conception and design: Lawton, Garcia. Acquisition of data: Garcia, Oh, Cole, Hendricks. Analysis and interpretation of data: Lawton, Garcia. Drafting the article: Lawton, Garcia. Critically revising the article: Lawton, Garcia. Reviewed submitted version of manuscript: all authors. Statistical analysis: Garcia. Administrative/technical/material support: Lawton. Study supervision: Lawton.

\section{Supplemental Information \\ Current Affiliations}

Dr. Garcia: Department of Neurological Surgery, Northwestern University, Chicago, IL.

\section{Correspondence}

Michael T. Lawton: c/o Neuroscience Publications, Barrow Neurological Institute, St. Joseph's Hospital and Medical Center, Phoenix, AZ.neuropub@barrowneuro.org. 